\title{
Habitat-induced reciprocal transformation in the root phenotype of Oriental ginseng is associated with alteration in DNA methylation
}

\author{
NGEZAHAYO Frédéric ${ }^{1 \dagger}$, WANG XiaoLi ${ }^{1,2 \dagger}$, YU XiaoMing $^{1}$, JIANG LiLi $^{1}$, CHU YunJie $^{3}$, \\ SHEN BaiHua ${ }^{4}$, YAN ZhongKai ${ }^{3} \&$ LIU Bao ${ }^{1 *}$ \\ ${ }^{1}$ Key Laboratory of Molecular Epigenetics of Ministry of Education, Northeast Normal University, Changchun 130024, China; \\ ${ }^{2}$ Department of Agronomy, Jilin Agricultural University, Changchun 130118, China; \\ ${ }^{3}$ Changchun University of Chinese Traditional Medicine, Changchun 130021, China; \\ ${ }^{4}$ Baihua Ginseng Cooperation Ltd, Shanghai 200010, China
}

Received September 23, 2010; accepted March 15, 2011

\begin{abstract}
Oriental ginseng is an important medicinal plant that grows in 2 major forms or ecotypes, wild and domesticated. Each form differs conspicuously in root phenotype, but can be converted from one type to another by habitat. Here we show that the habitat-induced transformation of ginseng root phenotype was accompanied by alteration in cytosine methylation at a large number of 5'-CCGG-3' sites detected by the methylation-sensitive polymorphism (MSAP) marker. The collective CG and CHG methylation levels of all 4 landraces of the domesticated form were significantly lower than those of the wild form. Interestingly, artificially transplanted ginseng plants recreated in both directions the methylation levels (at least in CHG) of their natural counterparts. The methylation differences between the 2 ginseng ecotypes were validated at 2 isolated MSAP loci bearing homology to a 5S rRNA gene or a copia retrotransposon. Our results implicate a link between epigenetic variation and habitat-induced phenotypic flexibility in Oriental ginseng.
\end{abstract}

cytosine methylation, epigenetics, habitat, Oriental ginseng, phenotypic flexibility

Citation: Ngezahayo F, Wang X L, Yu X M, et al. Habitat-induced reciprocal transformation in the root phenotype of Oriental ginseng is associated with alteration in DNA methylation. Chinese Sci Bull, 2011, 56: 1685-1690, doi: 10.1007/s11434-011-4484-1

Oriental ginseng (Panax ginseng C. A. Meyer) is a slowgrowing and long-lived perennial species with fleshy roots belonging to the Araliaceae [1]. Panax ginseng is the most renowned integrant in traditional Chinese medicine. Similar to its disjunctive counterpart species in the genus Panax, i.e, American ginseng (P. quinquefolius L.) [2], the biomedical functionality of an array of active compounds (primarily ginsenosides) extracted from ginseng roots has been documented in modern pharmacological and clinical investigations $[3,4]$.

Although the wild-form of $P$. ginseng has been used for more than 4000 years in China, its domestic cultivation

$\uparrow$ These authors contributed equally to this work.

*Corresponding author (email: baoliu@nenu.edu.cn) under an artificial canopy was started only about 300 years ago in the Changbai mountainous area in northeastern China, named the "Chang-Bai Mountain Man and Biosphere" (CBMMB) by the United Nations [5]. Nonetheless, the root phenotypes of wild and cultivated ginseng plants differ dramatically [6]. Interestingly, the morphology of ginseng roots is predominantly determined by the habitat in which the plants grow, and the typical root phenotypes of wild and domesticated forms can be reciprocally 'transformed' by growing seeds in, or transplanting 1- to 2-year-old seedlings to, a given habitat [5]. This is also largely true for American ginseng [7]. Thus, the ginseng root phenotype is remarkably flexible and represents a typical environment (habitat)-determined trait. This phenomenon has been unconsciously exploited by the ginseng farm- 
ers to produce "wild ginseng" roots from domesticated ginseng seeds and/or seedlings (for a better price) for thousands of years [5], as is the case for many other medicinal plants [8]. To our knowledge, the basis of this remarkable phenotypic flexibility in Oriental ginseng roots has not been investigated from a genetic or molecular perspective.

DNA cytosine methylation is a prominent epigenetic modification existing in many eukaryotes and is particularly abundant in plants [9-11]. Mounting evidence indicates that DNA methylation is sensitive, as well as responsive, to a sudden change in environmental conditions probably because of the physiological stresses they beget [12-17]. Such environment-induced alteration in DNA methylation may cause heritable, though potentially reversible, changes in gene expression and hence phenotypic novelty [14,18-20]. The aim of the present study was to explore whether the habitat-induced transformation of the Oriental ginseng root phenotype is associated with alterations in DNA methylation.

\section{Materials and methods}

\subsection{Plant materials}

Seven types of Oriental ginseng plants were used (Table S1), including: (i) wild ginseng (WG; 3 individuals); (ii) 4 major landraces of domesticated ginseng: Damaya (DG-DMY; 6 individuals), Yuanbangyuanlu (DG-YB; 6 individuals), Changbo (DG-CB; 6 individuals), and Shizhu (DG-SZ; 6 individuals); (iii) transplanted ginseng, consisting of domesticated ginseng (Damaya) transplanted to wild-ginseng habitat (natural forest canopy) for 20 years (TDG; 4 individuals) and wild ginseng transplanted to cultivated habitat (artificial canopy) for 6 years (TWG; 5 individuals). The wild-ginseng plants were collected from the CBMMB region, while the domesticated and transplanted ginseng plants were obtained from a ginseng garden (Helong Ginseng Farm) located inside CBMMB.

\subsection{Methylation-sensitive amplified polymorphism analysis}

Genomic DNA was isolated from fresh fibrous roots of each individual ginseng plant using the CTAB method. Methylation-sensitive amplified polymorphism (MSAP) [21] is a modified version of the standard amplified fragment polymorphism (AFLP) marker technique [22] by employing a pair of isoschizomers, Hpa II/Msp I, which is differentially sensitive to cytosine methylation at the $5^{\prime}$-CCGG-3' sites. Hpa II will not cut the sequence if either of the internal and external cytosines on the DNA strands is methylated, whereas Msp I will not cut the sequence if the external cytosine is hemimethylated [23]. Therefore, for a given DNA sample the full methylation of the internal cytosine (CG methylation) or hemimethylation of the external cytosine
(CHG methylation) at the sampled $5^{\prime}-\mathrm{CCGG}-3^{\prime}$ sites can be unequivocally assessed even in the presence of genetic variation $[21,24]$.

One pair of preselective primers and 40 pairs of selective primers were used for the MSAP analysis (Table S2). The amplicons were resolved by $5 \%$ denaturing polyacrylamide gel electrophoresis and visualized by silver staining [24]. Only clear and completely reproducible bands in 2 parallel MSAP experiments (starting from independent DNA extractions) were scored, and the levels of CG and CHG methylation for a given plant sample were calculated (Table S3). Typical methylation-variant bands were isolated and sequenced.

\subsection{Bisulfite sequencing}

Genomic DNA of each type of ginseng plant was modified by bisulfite using the EZ DNA Methylation-Gold Kit (Zymo Research, http://zymoresearch.com) in accordance with the manufacturer's instructions with minor modifications [25]. The amplification primer pairs for the 2 MSAP loci, MSAP16 (homologous to a ginseng 5S rRNA, GenBank accession No. EU234581) and MSAP37 (homologous to a low-copy ginseng LTR copia retrotransposon, GenBank accession No. EU046488; [26]), were designed using the Kismeth program (http://katahdin.mssm.edu/kismeth/). The primer sequences were: MSAP16-F (5'-GAAYTYYGAAGTTAAGYGTGYTT-3'), MSAP16-R (5'-CRRTRATTTARTRCTRRTATRATCRCA-3'), MSAP-37-F (5'-GAKTTRAAKGAAGAAATHTAYATGG-3') and MSAP37-R (5'-TARCAAYRRAATAACRAARTTWCCATC-3'); letters other than the 4 canonical nucleotide bases denote degenerate bases, i.e, H (A/T/C), K (T/G), R (A/G), W (A/T) and Y $(\mathrm{C} / \mathrm{T})$. The PCR products were cloned and sequenced. For locus MSAP16 (the 5S rRNA gene), considering its high-copy nature, 30 random clones for each sample were sequenced, while for locus MSAP37 15 random clones for each sample were sequenced. The methylation level, expressed as percentage (\%) per site for each of the three types of cytosines, $\mathrm{CG}, \mathrm{CHG}$ and $\mathrm{CHH}$, was calculated by dividing the number of non-converted (methylated) cytosines by the total number of cytosines at a given position.

\section{Results and discussion}

The difference in $P$. ginseng root phenotypes between the studied wild and domesticated forms was clear (Figure 1), consistent with the established criteria to differentiate the two forms ([5]; Table S1). Remarkably, when the one-yearold seedlings of domesticated ginseng (landrace Damaya) were transplanted to the WG-growing habitat and allowed to grow for 20 years, their roots exhibited the phenotype resembling wild-ginseng (WG). Similarly, when seedlings of wild-ginseng plants were grown under the domesticcated- 

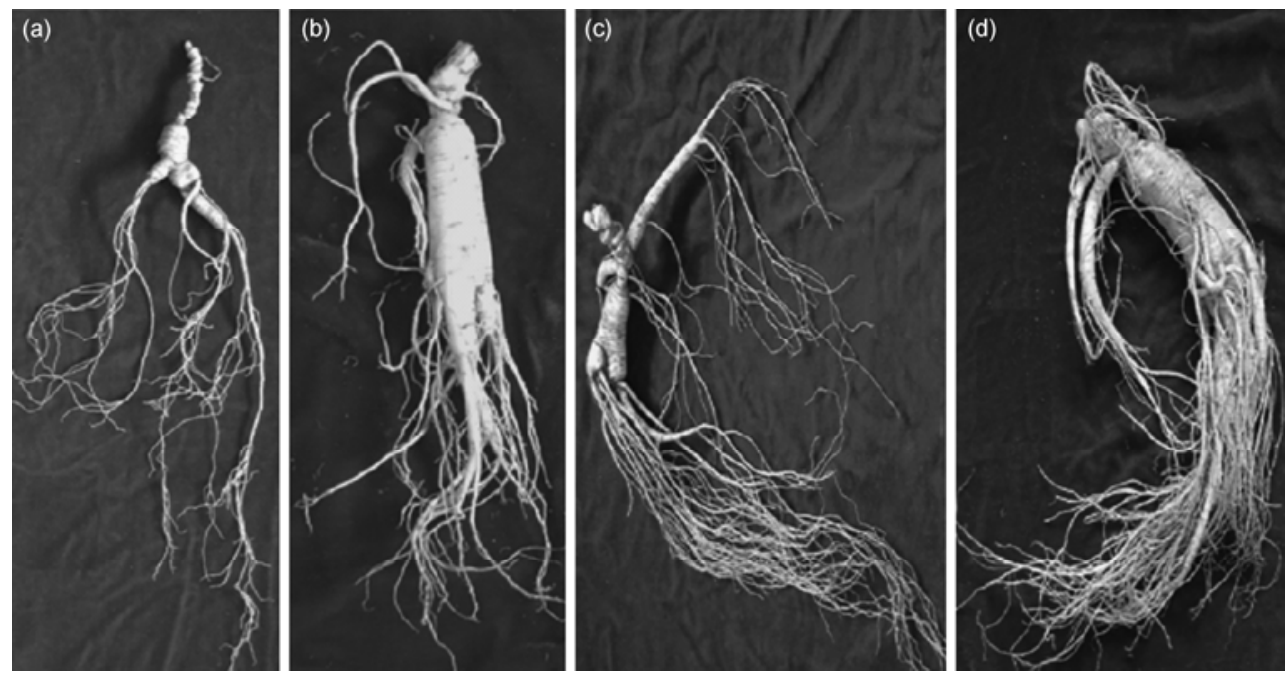

Figure 1 Typical root phenotypes of Oriental ginseng (Panax ginseng) of the 4 groups of plants. (a) A wild ginseng individual grown uncovered in the natural habitat in the CBMMB region in northeast China; the plant age was ascertained to be $c a .30$ years; (b) a domesticated ginseng (landrace Damaya) individual grown for 6 years under cultivation; (c) a domesticated ginseng (landrace Damaya) individual transplanted to and grown for 20 years in the wild-ginseng habitat in the CBMMB region; (d) a 6-year-old wild-ginseng plant grown under cultivation starting from seed germination.

ginseng cultivation condition for 6 years (TWG), the plants showed the root phenotype of domesticated ginseng.

We explored the possibility that this remarkable habitat-induced phenotypic flexibility in the Oriental ginseng root is linked to differential epigenetic modifications by DNA cytosine methylation. We employed MSAP markers [21] to assess the genome-wide methylation levels of two major sequence types, $\mathrm{CG}$ and $\mathrm{CHG}$, at the 5'-CCGG-3' sites (the prominent sites for DNA methylation modification in eukaryotes).

Using 40 pairs of selective primers (Table S2), we scored $>2000$ MSAP loci across the ginseng genome (Table S3). By analyzing the data we obtained the following observations: (i) significant differences in CG and CHG methylation levels existed among the 4 domesticated ginseng landraces (DG-DMY, DG-YBYL, DG-CB and DG-SZ), with DG-DMY showing the highest level and DZ-CB showing the lowest level (Figure 2(a); Table S3); (ii) relative to WG, the $\mathrm{CG}$ and $\mathrm{CHG}$ methylation levels of all 4 major domesticated landraces, when considered collectively, were substantially decreased (Figure 2(a); Table S3); and (iii) intriguingly, TWG manifested significantly lower CHG methylation levels than WG, but no change in CG levels, while the transplanted domesticated plants (TDG) showed increased methylation compared with DG-YBYL, DG-CB and DG-SZ (Figure 2(a); Table S3). The latter observation suggested that $\mathrm{CHG}$ methylation was more sensitive to abrupt changes in habitat and may undergo rapid alterations accordingly. Typical MSAP bands showing methylation alterations in response to habitat alteration were isolated, sequenced and subjected to a BLAST query against the GenBank databases. Out of 32 analyzed MSAP bands, we identified 17 bands that bore meaningful homologies to protein-coding genes, ribosomal RNA genes and mobile genetic elements (Table S4).

To validate the methylation alterations in a locus-specific manner, we bisulfite-sequenced 2 MSAP loci, MSAP16 (263 bp) and MSAP37 (247 bp), which bore homologies to a ginseng $5 \mathrm{~S}$ rRNA-coding gene and a low-copy ginseng copia retrotransposon, respectively ([26]; Table S4).

The bisulfite-sequencing results suggested that, although in general the ginseng types did not manifest clear differences in cytosine methylation levels at these two specific loci, statistical analysis indicated that the following 2 sets of comparisons were significantly different: (i) for MSAP16, the level of CHG methylation was significantly lower $(P<$ 0.05 ) in the 4 domesticated ginseng landraces (considered together) than in WG (Figure 2(b)); (ii) for MSAP37, the methylation level of all three cytosine types (CG, CHG and $\mathrm{CHH})$ was significantly lower $(P<0.05$ or 0.01$)$ in the 4 domesticated ginseng landraces (considered together) than in WG (Figure 2(c)). We noted that for these 2 loci, the methylation levels of all 3 cytosine types did not show a significant difference $(P>0.05)$ between the transplants and their respective non-transplanted counterparts (Figure 2(b) and (c); data not shown), which appears to differ from the significant increase in $\mathrm{CHG}$ methylation detected by the more global, MSAP-based method (Figure 2(a)). The incongruence between the MSAP and bisulfite-sequencing data can be readily reconciled by the following 2 considerations: (i) DNA methylation alteration did not occur in all 5'-CCGG-3' loci for all cytosine types (CG, CHG and $\mathrm{CHH}$; (ii) while for some genomic loci the alteration might occur rapidly in response to the habitat shift, for other loci (e.g., the 2 analyzed in this study) a time-frame longer than that experienced by the transplants is probably required for methylation of any site to undergo a significant change.

The short history of Oriental ginseng domestication (ca. 

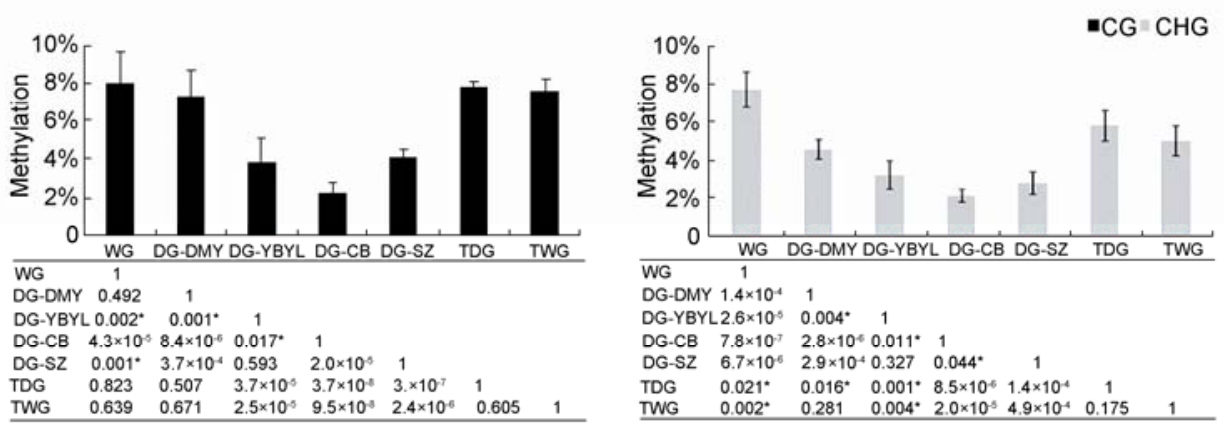

(a)
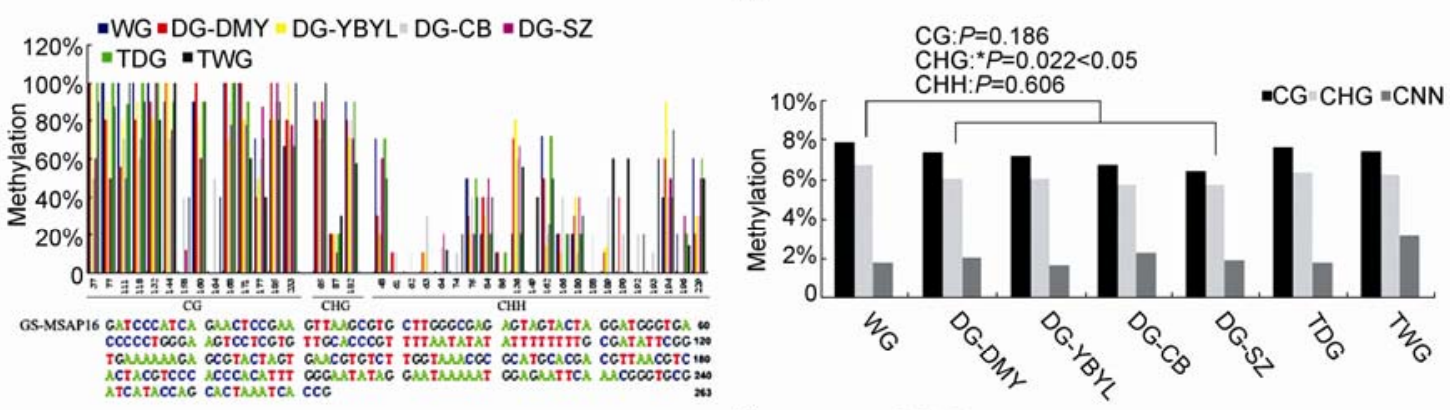

(b)
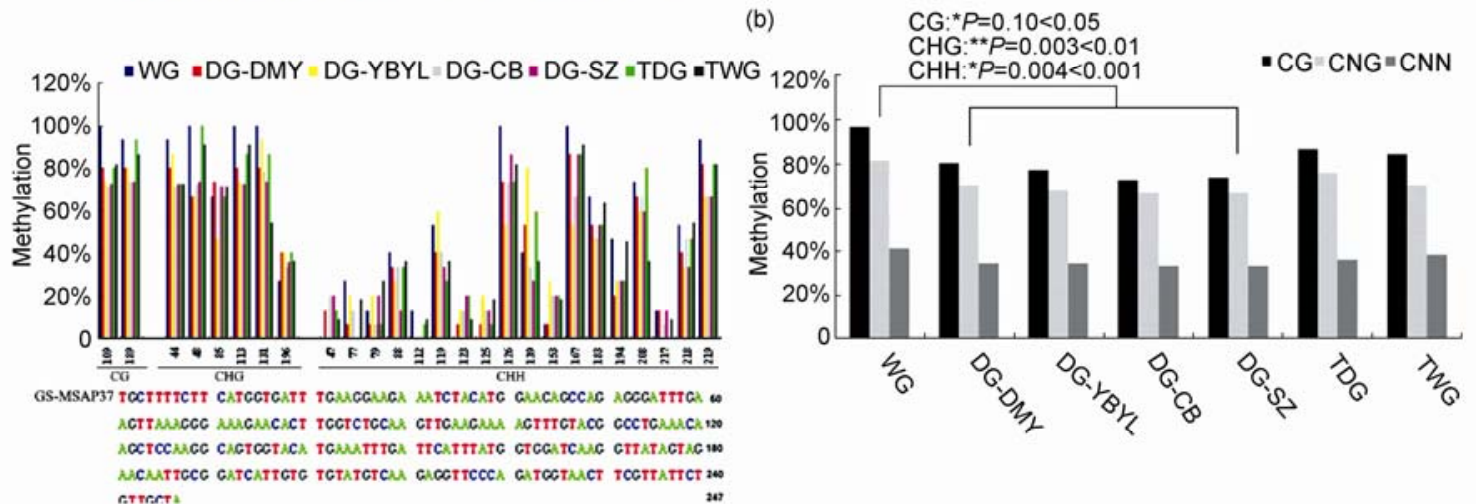

(c)

Figure 2 (a) MSAP-based assessment of genome-wide CG and CHG methylation levels at the 5'-CCGG-3' sites in the different types of Oriental ginseng comprising: wild-ginseng (WG); 4 landraces of domesticated ginseng, Damaya (DMY), Yuanbangyuanlu (YBYL), Changbo (CB), and Shizhu (SZ); transplanted domesticated ginseng Damaya (TDG); and transplanted wild ginseng (TWG). (b) and (c) Bisulfite sequencing-based cytosine methylation maps of two selected MSAP bands, MSAP16 (b) and MSAP37 (c). Relative methylation levels (\%) of each of the 3 types of cytosines (CG, CHG and CHH) are given both at each position (left) and on a collective basis (right). The numbers on the $x$-axis denote the cytosine positions. The sequences of both analyzed MSAP bands are presented. Statistical significance (based on Student's $t$-test) between the comparisons is indicated by an asterisk at the 0.05 statistical level or two asterisks at the 0.01 statistical level.

300 years) coupled with a lack of judicious selection and hybridization-based breeding probably has not yet allowed the domesticated landraces to become genetically distinct from their wild progenitors. This is likely also the situation for American ginseng, as implied in a previous study [27]. This might explain why intensive efforts to authenticate cultivated and wild forms by a variety of DNA markers at the intraspecific level have met with limited success [28-31]. Our results indicate that the 2 forms of ginseng are nonetheless epigenetically distinct if grown under different habitats. This was illustrated by the fact that: (i) the wild and domesticated forms of Oriental ginseng sampled from distinct habitats possessed markedly different cyto- sine methylation levels both on a global scale (MSAP) and in a locus-specific manner (bisulfite-sequencing); (ii) the cytosine methylation state for a substantial portion of genomic loci of CHG sites (based on MSAP) is reversible with alteration of habitat, which coincided with transformation of the root phenotype in the reciprocally transplanted plants. Though observational, our findings implied partitioning of environment-induced epigenetic alterations in the habitat-dependent phenotypic flexibility of ginseng roots, probably via methylation-regulated changes in gene expression.

The mechanism underlying the habitat-induced cytosine methylation alteration in ginseng remains elusive. One pos- 
sible scenario might be that under cultivation ginseng plants grow much faster as a result of enriched nutrient levels and a reduced canopy, perhaps promoting more rapid cell divisions, but these might not be accompanied by timely enhanced expression and/or activity of the DNA methyltransferases essential for perpetuating the methylation status; conceivably, this will result in progressive loss of cytosine methylation from many genomic loci in a passive manner [32]. Although demethylation at some cytosines can to some extent be antagonistically corrected by the small RNA machinery [33], it is conceivable that demethylation at other sites might remain uncorrected. Alternatively, the sudden change in habitat might cause a general disruption of the established physiological homeostasis in WG plants and, for successive acclimation to a new habitat, epigenetic remodeling is mechanistically essential. Further experiments are required to investigate these possibilities, which might provide novel clues towards more targeted manipulation of ginseng morphological, physiological and pharmaceutical phenotypes, probably through induced changes in the ginseng epigenome.

This work was supported by the National Natural Science Foundation of China (30870178).

1 Wen J, Zimmer E A. Phylogeny and biogeography of Panax L. (the Ginseng genus, Araliaceae): Inferences from ITS sequences of nuclear ribosomal DNA. Mol Phylogenet Evol, 1996, 6: 167-177

2 Zhu S, Fushimi H, Cai S, et al. Phylogenetic relationship in the genus Panax: Inferred from chloroplast trnK gene and nuclear 18S rRNA gene sequences. Planta Med, 2003, 69: 647-653

3 Goldrosen M H, Straus S E. Complementary and alternative medicine: Assessing the evidence for immunological benefits. Nat Rev Immunol, 2004, 4: 912-921

4 Jia L, Zhao Y, Liang X. Current evaluation of the millennium phytomedicine-Ginseng (II): Collected chemical entities, modern pharmacology, and clinical applications emanated from traditional Chinese medicine. Curr Med Chem, 2009, 16: 2924-2942

5 Zhang S C. The Chinese Ginseng. Shanghai: The Shanghai S \& T Publishing House, 1992

6 Goldstein B. Ginseng: Its history, dispersion, and folk tradition. Am J Chinese Med, 1975, 3: 223-234

7 Nadeau I, Olivier A. The biology and forest cultivation of American ginseng (Panax quinquefolius L.) in Canada. Can J Plant Sci, 2003, 83: 877-891

8 Canter P H, Thomas H, Ernst E. Bringing medicinal plants into cultivation: Opportunities and challenges for biotechnology. Trends Biotechnol, 2005, 23: 180-185

9 Martienssen R A, Colot V. DNA methylation and epigenetic inheritance in plants and lamentous fungi. Science, 2001, 293: 1070-1074

10 Cao X, Jacobsen S E. Locus-specific control of asymmetric and CpNpG methylation by the DRM and CMT3 methyltransferase genes. Proc Natl Acad Sci USA, 2002, 99: 16491-16498

11 Cao X, Aufsatz W, Zilberman D, et al. Role of the DRM and CMT3 methyltransferases in RNA-directed DNA methylation. Curr Biol, 2003, 13: 2212-2217

12 Boyko A, Kovalchuk I. Epigenetic control of plant stress response. Environ Mol Mutagen, 2008, 49: 61-72

13 Chinnusamy V, Zhu J K. Epigenetic regulation of stress responses in plants. Curr Opin Plant Biol, 2009, 12: 133-139

14 Adams K L. Dandelions 'remember' stress: Heritable stress-induced methylation patterns in asexual dandelions. New Phytol, 2010, 185: 867-868

15 Gao L, Geng Y, Li B, et al. Genome-wide DNA methylation alterations of Alternanthera philoxeroides in natural and manipulated habitats: Implications for epigenetic regulation of rapid responses to environmental fluctuation and phenotypic variation. Plant Cell Environ, 2010, 33: 1820-1827

16 Verhoeven K J F, Jansen J J, van Dijk P J, et al. Stress-induced DNA methylation changes and their heritability in asexual dandelions. New Phytol, 2010, 185: 1108-1118

17 Zhang M, Kimatu J N, Xu K, et al. DNA cytosine methylation in plant development. J Genet Genomics, 2010, 37: 1-12

18 Varmuza S. Epigenetics and the renaissance of heresy. Genome, 2003, 46: 963-967

19 Lukens L N, Zhan S. The plant genome's methylation status and response to stress: Implications for plant improvement. Curr Opin Plant Biol, 2007, 10: 317-322

20 Rapp R A, Wendel J F. Epigenetics and plant evolution. New Phytol, 2005, 168: 81-91

21 Reyna-López G E, Simpson J, Ruiz-Herrera J. Differences in DNA methylation patterns are detectable during the dimorphic transition of fungi by amplification of restriction polymorphisms. Mol Genet Genomics, 1997, 253: 703-710

22 Vos P, Hogers R, Bleeker M, et al. AFLP: A new technique for DNA fingerprinting. Nucleic Acids Res, 1995, 23: 4407-4414

23 McClelland M, Nelson M, Raschke E. Effect of site-specific modification on restriction endonucleases and DNA modification methyltransferases. Nucleic Acids Res, 1994, 22: 3640-3659

24 Wang Y M, Dong Z Y, Zhang Z J, et al. Extensive de novo genomic variation in rice induced by introgression from wild rice (Zizania latifolia Griseb.). Genetics, 2005, 170: 1945-1956

25 Ngezahayo F, Xu C, Wang H, et al. Tissue culture-induced transpositional activity of mPing is correlated with cytosine methylation in rice. BMC Plant Biol, 2009, 9: 91

26 Bai D, Brandle J, Reeleder R. Genetic diversity in North American ginseng (Panax quinquefolius L.) grown in Ontario detected by RAPD analysis. Genome, 1997, 40: 111-115

27 Liu X D, Zhong X F, Ma Y, et al. Copia retrotransposons of two disjunctive Panax species: $P$. ginseng and $P$. quinquefolius. Aus J Bot, 2008, 56: 177-186

28 Ngan F, Shaw P, But P, et al. Molecular authentication of Panax species. Phytochemistry, 1999, 50: 787-791

29 Zhang Y, Shaw P, Saze C, et al. Molecular authentication of Chinese herbal materials. J Food Drug Anal, 2007, 15: 1-9

30 Jo B, Suh D, Cho E, et al. Characterization of polymorphic microsatellite loci in cultivated and wild Panax ginseng. Genes Genomics, 2009, 31: 119-127

31 Kwon H K, Ahn C H, Choi Y E. Molecular authentication of Panax notoginseng by specific AFLP-derived SCAR marker. J Med Plant Res, 2009, 3: 955-964

32 Saze H. Epigenetic memory transmission through mitosis and meiosis in plants. Semin Cell Dev Biol, 2008, 19: 527-536

33 Teixeira F K, Heredia F, Sarazin A, et al. A role for RNAi in the selective correction of DNA methylation defects. Science, 2009, 323: 1600-1604

Open Access This article is distributed under the terms of the Creative Commons Attribution License which permits any use, distribution, and reproduction in any medium, provided the original author(s) and source are credited. 